\title{
Intermédialités
}

Histoire et théorie des arts, des lettres et des techniques

Intermediality

History and Theory of the Arts, Literature and Technologies

\section{Aspects philosophiques de la disparition : un détour par la Chine ancienne}

\section{Nicolas Zufferey}

Numéro 10, automne 2007

Disparaître

Disappearing

URI : https://id.erudit.org/iderudit/1005552ar

DOI : https://doi.org/10.7202/1005552ar

Aller au sommaire du numéro

Éditeur(s)

Centre de recherche sur l'intermédialité

ISSN

1705-8546 (imprimé)

1920-3136 (numérique)

Découvrir la revue

Citer cet article

Zufferey, N. (2007). Aspects philosophiques de la disparition : un détour par la Chine ancienne. Intermédialités / Intermediality, (10), 55-60.

https://doi.org/10.7202/1005552ar
Résumé de l'article

En Chine ancienne, le vocabulaire pour dire la disparition n'est pas celui de l'annihilation, c'est celui de l'éclipse, de l'oubli, du déplacement, éventuellement de la réduction ou de la transformation. La disparition est avant tout affaire de mouvement ou de déplacement : les choses disparaissent parce qu'elles sont passées dans une autre dimension, parce qu'elles ne coïncident plus dans l'espace, ou parce qu'elles sont cachées; elles n'en continuent pas moins d'exister. La mort elle-même s'explique par le mouvement, plus précisément par un réagencement de matière; le mort se transforme en autre chose, redevient matière brute - à proprement parler, il n'est pas anéanti. En Chine ancienne, la disparition n'est donc qu'un aspect de l'infinie variation des choses et du monde. 


\title{
Aspects philosophiques de la disparition: un détour par la Chine ancienne
}

\author{
Nicolas Zufferey
}

Au bord du fleuve, les gens vont et viennent, Ne pensant qu'à déguster la perche délicieuse.

Mais avez-vous remarqué cette pauvre voile Qui apparaît et disparaît dans les vagues et le vent ${ }^{1}$ ?

Un pêcheur dans le fleuve (Fan Zhongyan, 989-1052)

tymologiquement, notre mot «disparaître » renvoie à la phénoménologie: « disparaît» ce qui est privé (dis-) de paraître, et donc n'apparaît plus. De ce point de vue, la vision chinoise n'a rien d'étonnant. En Chine ancienne et impériale ${ }^{2}$, la disparition est plus affaire de phénoménologie que d'ontologie, ou tout au moins, elle n'a pas toujours des implications ontologiques radicales: disparaître, ce n'est pas forcément être anéanti, ce n'est pas perdre son être ou son existence, c'est simplement ne plus être visible ou ne plus être vu. Le vocabulaire chinois pour dire le disparaître n'est pas celui de l'annihilation, c'est celui de l'éclipse, de l'oubli, du déplacement, éventuellement de la réduction ou de la transformation. Dans notre traduction du poème, cité en exergue, la voile apparaît et disparaît», dans le texte chinois, elle se contente de «sortir (chu) et

1. Notre traduction. Pour le texte chinois de ce poème relativement peu connu, voir Song shi jianshang cidian, Shanghai, Shanghai cishu chubanshe, 1987, p. 59.

2. Les sources citées dans cet article datent de l'époque des Royaumes Combattants (475-221 av. J.-C.), c'est-à-dire de la fin de l'époque ancienne (Ancient China) - qui se termine en 221 av. J.-C. avec l'unification impériale - et de l'époque impériale jusqu'au $\mathrm{XIV}^{\mathrm{e}}$ siècle. Cette dernière période correspond approximativement au concept de Early Imperial China, défini d'ailleurs de manière variable selon les auteurs. 
sombrer (mo) » : le mot composé des deux caractères chu et mo (chumo), suggère, encore en chinois moderne, l'alternance entre ce qui est visible et ce qui ne l'est pas, entre le présent et l'absent. La barque, lorsqu'elle est au creux de la vague, est soustraite au regard, mais elle n'est pas soustraite au monde: dans un instant, elle réapparaîtra. Notre barque disparaît parce qu'elle est cachée. On pourrait dire aussi que la disparition résulte du passage de la barque dans une autre dimension que celle de l'observateur, dimension à laquelle celui-ci, au moins durant un court instant, n’a pas accès. De façon générale, en Chine ancienne, le passage à une autre dimension n'implique ni anéantissement, ni coupure radicale, et ce même lorsque la dimension en question est celle des morts. Dans le texte suivant, tiré de la très officielle Histoire dynastique des Han postérieurs (compilée au $v^{e}$ siècle de notre ère), Liu Gen, un magicien de la fin de la dynastie Han (2o6 av. J.-C.-220 apr. J.-C.) fait apparaître des défunts:

Liu Gen s'était fait ermite dans les monts Song, et ses adeptes venaient de très loin pour étudier la Voie. Le préfet Shi Qi, qui jugeait que Liu Gen trompait les gens avec sa prétendue sorcellerie, le fit arrêter et conduire au siège de la commanderie, où il le réprimanda ainsi: "Sur la base de quelle technique secrète te permets-tu de mentir et de duper ainsi les gens? Si tu as vraiment des talents magiques, il faut me le prouver sur-le-champ, sinon je te ferai immédiatement exécuter»! Liu Gen répondit: «Je n’ai pas de talent extraordinaire, si ce n'est que je suis capable de rendre visible les esprits.» Le préfet fit: «Dépêche-toi de les appeler, que je les voie de mes propres yeux, et je serai convaincu!» Liu Gen regarda alors à gauche, en sifflant, et au bout d'un moment, les parents et ancêtres décédés de Shi Qi, ainsi que d'autres membres de sa famille proche, soit plusieurs dizaines de personnes, s'avancèrent, mains liées dans le dos. Ils se prosternèrent devant Liu Gen: "Notre rejeton manque totalement d'éducation, il mérite mille fois la mort!» Puis ils se retournèrent vers Shi Qi pour le houspiller: «Toi, notre descendant, non seulement tu ne nous es d'aucune utilité, mais tu attires sur les âmes des défunts les ennuis et les humiliations! Prosterne-toi vite pour nous faire pardonner! Effrayé et désolé, Shi Qi se jeta sur le sol, le frappant de son front jusqu'au sang, et se disant prêt à mourir pour racheter sa faute. Liu Gen maugréa sans répondre, et d'un coup tous s'en allèrent on ne sait où̉.

Dans ce texte, le magicien ne ressuscite pas les morts, il se contente de les faire apparaître : ceux qui avaient disparu (les défunts) n’avaient pas été anéantis, mais transportés dans une autre réalité. Le magicien rend visible ce qui est invisible aux gens ordinaires; la magie, ici, est de nature médiumnique, elle fait communiquer des sphères qui sont normalement séparées. À la fin de l'épisode,

3. Hou Hanshu, chap. 82, Pékin, Zhonghua shuju, vol. 10, p. 2746 (notre traduction). 
les morts s'en vont, mais là encore, les mots pour décrire cette disparition n'ont pas de connotation ontologique: les défunts s'en vont, et seule la soudaineté de leur départ («d'un coup tous s'en allèrent») indique le surnaturel.

Le médium fait coïncider deux ordres normalement séparés. On touche à l'idée de coïncidence, qui est instructive pour mieux appréhender les conceptions implicites sur la disparition en Chine ancienne : disparaît ce qui ne coïncide plus dans l'espace avec l'observateur, généralement en raison d'un mouvement ou d'un déplacement. À l'inverse, pour que les choses s'apparaissent les unes aux autres, il faut qu'elles «tombent ensemble». La coïncidence implique parfois une part de chance ou de hasard, et les auteurs chinois ont largement usé de ce ressort. Dans le roman chinois traditionnel, les personnages sont nombreux, et ils apparaissent et disparaissent au fil du récit, dont la trame est souvent lâche, au gré de la fantaisie du conteur ou du narrateur. Le célèbre roman Au bord de l'eau (XIV siècle), qui raconte les tribulations de 108 brigands, recourt de manière fréquente à ce procédé4. Au chapitre 6, par exemple, Lu Da, l'un des brigands, erre sans but dans la forêt des Pins rouges, qui n'est pas localisée de manière précise, mais se situe à l'est de la Chine (sans doute aux confins des actuelles provinces du Shandong et du Henan). Dans cette forêt, il tombe totalement par hasard sur Shi Jin, un autre des brigands, qu'il a quitté bien des mois plus tôt à Weizhou (dans l'actuel Gansu), à l'extrémité occidentale de l'Empire. À vol d'oiseau, il y a plus de mille kilomètres entre les deux lieux. De cette rencontre improbable, les deux comparses s'étonnent à peine: "Qui aurait dit que nous nous retrouverions? " (ABL, p. 147), demande simplement l'un d'eux. La désinvolture avec laquelle le narrateur réunit ses personnages fait écho à celle dont il fait preuve pour les faire disparaître. Au début du chapitre 3, le brigand Shi Jin était le héros du récit; mais à un moment, il « rentre à son hôtellerie » — et disparaît du récit: ce sont d'autres personnages qui prennent le relais. Après leur rencontre du chapitre 6, Shi Jin et Lu Da partagent quelques aventures (ABL, p. 147-152), puis se séparent à nouveau. À nouveau, c'est Shi Jin qui disparaît: «Laissons Shi Jin », dit sobrement le texte, sans autre justification. Mais si Shi Jin a disparu, il n'est pas mort pour autant: «Nous nous retrouverons bien un de ces jours» (ABL, p. 151), dit-il d'ailleurs à Lu Da; et effectivement, Shi Jin réapparaît plus tard dans le roman.

4. Shi Nai-an et Luo Guan-zhong, Au Bord de l'eau, trad. Jacques Dars, Paris, Gallimard, coll. «Bibliothèque de la Pléiade», 2 vol., 1978. Désormais, les références à cet ouvrage seront indiquées par le sigle «ABL », suivi de la page, et placées entre parenthèses dans le corps du texte. 
Apparaître et disparaître est donc ici simple affaire de coïncidence (dans tous les sens du terme). Notons la proximité entre le médium qui fait apparaître les morts, et le romancier (le narrateur) qui retrouve un personnage: on parlera de magie ou de surnaturel dans le premier cas, de hasard dans le deuxième, mais à chaque fois il s'agit de rendre visible en tirant de l'oubli et de faire (re-)coïncider des personnages avec un observateur, que celui-ci soit préfet ou simple lecteur de roman.

Pour en revenir à la magie, la jolie histoire suivante, également tirée de l'Histoire des Han postérieurs, est instructive dans notre contexte. Le magicien Zuo le Bienveillant est capable de faire apparaître à volonté biens et nourritures, mais il se heurte à la suspicion du grand homme d'État Cao Cao (155-220):

Cao Cao se rendit dans les faubourgs de la capitale, accompagné de ses grands officiers et serviteurs, soit une suite d'environ cent personnes. [Utilisant sa magie] Zuo le Bienveillant présenta à chacune d'elles une grande coupe de vin et une livre de viande séchée: il leur versa lui-même à boire, et chacun fut rassasié. Cao Cao, étonné, ordonna une enquête: on inspecta les boutiques du marché, et il s'avéra que toutes avaient été vidées de leur vin et viande. Mécontent, Cao Cao fit appréhender Zuo le Bienveillant en plein banquet pour le mettre à mort; mais Zuo le Bienveillant pénétra dans le mur, et d'un coup disparut. Quelqu'un l'aperçut sur le marché, et on essaya à nouveau de le capturer; mais toutes les personnes sur le marché se transformèrent, prenant l'apparence de Zuo le Bienveillant, si bien qu'on ne savait plus qui était qui. Par la suite, on le rencontra au sommet des monts Yangcheng [dans l'actuel Henan] : à nouveau, on le poursuivit, mais il se glissa dans un troupeau de moutons. Cao Cao, comprenant qu'il ne pourrait pas l'attraper, commanda de lui dire la chose suivante: « Mon but n'est pas de vous mettre à mort, je voulais simplement mettre à l'épreuve vos pouvoirs magiques. Soudain, un vieux bélier, pliant ses pattes antérieures, se dressa [sur ses membres postérieurs] à la manière d'un homme et s'écria: «Pourquoi m’effrayer de la sorte?». Les hommes de Cao Cao se précipitèrent pour l'attraper, mais alors tous les moutons, soit plusieurs centaines, se transformèrent en béliers, et dressés comme des humains, les pattes avant repliées, s'écrièrent: «Pourquoi m'effrayer de la sorte?» Si bien qu'on ne savait pas qui attraper ${ }^{5}$.

Ce récit est d'autant plus extraordinaire qu'il est tiré d'une histoire dynastique officielle. Sous cette forme, le texte date du ve siècle de notre ère, mais il fait référence à des personnages de la fin du $\mathrm{II}^{\mathrm{e}}$ siècle, époque très troublée, et une interprétation possible est politique. Nous n'explorerons pas cette piste ici. Nous nous contenterons de souligner deux traits révélateurs dans notre contexte. Tout d'abord, lorsque Zuo le Bienveillant fait apparaître du vin et de la viande pour une centaines de convives, il ne crée rien : il s'est contenté de déplacer vers lui (ou d'aller chercher), par magie, les aliments qui se trouvaient dans les échoppes du

5. Hou Hanshu, chapitre 82, p. 2747-2748 (notre traduction). 
marché. Rien ne se crée, rien ne se perd: ce qui a disparu là-bas reparaît ici. De la même façon, lorsque Zuo le Bienveillant disparaît pour fuir ses poursuivants, il reparaît ailleurs, éventuellement sous une autre forme: il continue à exister. Autre trait instructif, la transformation: notre magicien se soustrait à ses ennemis grâce à des métamorphoses. Tous les clients du marché prennent son apparence, puis il se transforme en bélier: en un sens, le magicien disparaît, puisqu'on ne le voit plus sous son apparence normale, mais bien évidemment, il continue à exister.

Déplacement et transformation ont deux aspects en commun. Premièrement, bien sûr, dans les deux cas, il n'y a pas de cessation de l'existence: en termes modernes, on parlera peut-être de préservation de la matière. Et deuxièmement, le déplacement comme la transformation impliquent un mouvement. Cela est essentiel pour comprendre la nature de la disparition en Chine ancienne: les choses et les êtres disparaissent parce qu'ils bougent et changent sans cesse, et la disparition n'est qu'un aspect de l'infinie variation des choses et du monde.

La mort elle-même, pour les lettrés de la Chine ancienne, est moins destruction que simple transformation, c'est-à-dire mouvement de matière. On a rencontré ci-dessus des défunts qui surgissent d'une autre dimension sous la forme de fantômes, mais pour les élites lettrées, moins crédules, la mort est plus terre à terre: elle n'est pas passage d'une dimension à l'autre (du monde des vivants au monde des morts), mais simplement d'un état à un autre. Dans les mots du célèbre philosophe taoïste Zhuangzi (Ive siècle av. J.-C.) :

La femme de Zhuangzi étant morte, [son ami] Hui Shi s'en fut lui offrir ses condoléances. Il trouva Zhuangzi négligemment assis les jambes écartées et chantant en battant la mesure sur une écuelle. Hui Shi lui dit:

- Que vous ne pleuriez pas la mort de celle qui fut la compagne de votre vie et qui éleva vos enfants, c'est déjà assez, mais que vous chantiez en battant l'écuelle, c'est trop fort!

- Du tout, dit Zhuangzi. Au moment de sa mort, je fus naturellement affecté un instant, mais réfléchissant sur le commencement, je découvris qu'à l'origine elle n'avait pas de vie; non seulement elle n'avait pas de vie, mais pas même de forme; non seulement pas de forme, mais pas même de souffle. Quelque chose de fuyant et d'insaisissable se transforme en souffle, le souffle en forme, la forme en vie, et maintenant voici que la vie se transforme en mort. Tout cela ressemble à la succession des quatre saisons de l'année. En ce moment, ma femme est couchée tranquillement dans la Terre. Si je me lamentais en sanglotant bruyamment, cela signifierait que je ne comprends pas le cours du Destin. C'est pourquoi je m'abstiens ${ }^{6}$.

6. Zhuangzi, L'œuvre complète de Tchouang-tseu, chap. 18, , trad. Liou Kia-hway, Paris, Éditions Gallimard, coll. «Collection UNESCO d'œuvres représentatives. Série chinoise. Connaissance de l'Orient», 1969, p. 145-146. 
Wang Chong, le grand penseur du I ${ }^{\text {er }}$ siècle de notre ère, résume fort bien les choses dans ses Discussions critiques:

Au plus fort de l'hiver, ce sont les fluides glacés qui l'emportent, et l'eau se cristallise pour devenir de la glace. Au printemps, les fluides s'adoucissent, et la glace fond. La vie humaine est un peu comme la glace: les fluides yin et yang se cristallisent et donnent les êtres humains; une fois leur vie achevée, ils meurent et redeviennent fluides?

En bref, pour les penseurs chinois, qu' ils soient taoïstes comme Zhuangzi, ou plutôt proches du confucianisme comme Wang Chong, rien ne disparaît jamais entièrement: les choses se déplacent ou se transforment, elles paraissent disparâ̂tre parce que la matière est en perpétuel mouvement, que ce soit dans le temps ou l'espace, mais elles ne s'anéantissent pas. Le changement est au cœur de l'être.

A l'inverse, et en simplifiant, les modèles de type platonicien ou chrétien, qui ont privilégié l'immuable ou l'éternel (les Idées, l'âme, Dieu), laissent moins de place pour les transitions, les transformations, les états intermédiaires: les essences étant en quelque sorte absolues, irréductibles, la seule alternative à l'existence, c'est la non-existence, le non-être, la disparition radicale ou, bien sûr, le passage dans une transcendance par définition séparée et inaccessible ${ }^{8}$. En d'autres termes, en occident, la disparition prend plus facilement un tour ontologique, définitif, que ça n’est le cas dans le modèle chinois. Tout cela est bien sûr une simplification, et surtout n'implique pas que la disparition d'un être cher soit plus facile à vivre pour un Chinois que pour un occidental, et cela d'autant moins que la distinction entre corps et âme ayant joué un rôle secondaire dans la culture chinoise, les Chinois n'ont guère eu la consolation d'imaginer qu'ils retrouveraient leurs disparus dans l'au-delà.

7. Lunheng, 62.10, Lunheng zhushi, Pékin, Zhonghua shuju, 1979, vol. 3, p. 1196 (notre traduction).

8. Pour une vision (trop) tranchée de l'opposition entre pensée chinoise et pensée occidentale en l'espèce, voir par exemple François Jullien, Procès ou création: une introduction à la pensée des lettrés chinois. Essai de problématique interculturelle, Paris, Seuil, coll. «Des travaux», 1989. Nous ne pouvons examiner ici la façon dont les anciens Chinois envisagent l'être (au sens ontologique); rappelons simplement que nos concepts d'«être » et de «non-être» n'ont pas de véritable équivalent dans la tradition philosophique chinoise. Un couple approchant serait you/wu, qu'on rendra cependant en français par «(y) avoir » / «ne pas (y) avoir» plutôt qu'au moyen du verbe être; Voir Angus C. Graham, «Being in Western Philosophy Compared with shi/fei and wu/you in Chinese Philosophy ", Asia Major (nouvelle série), vol. 7, n 10 1-2, 1959, p. 79-112. Ces différences pèsent évidemment d'un grand poids sur les conceptions respectives quant à la question de la disparition dans les deux traditions. 